\title{
Mast cell deficiency attenuates acupuncture analgesia for mechanical pain using c-kit gene mutant rats
}

\author{
Xiang Cui,, ,2,* Kun Liu, 1,* \\ Dandan Xu, ${ }^{1,3}$ Youyou Zhang, ${ }^{1,4}$ \\ Xun He,' Hao Liu, ${ }^{1,5}$ Xinyan \\ Gao,' Bing Zhu' \\ 'Department of Physiology, Institute of \\ Acupuncture and Moxibustion, China \\ Academy of Chinese Medical Sciences, \\ Beijing, China; ${ }^{2}$ College of Acupuncture \\ and Orthopedics, Hubei University of \\ Chinese Medicine, Wuhan, China; ${ }^{3} \mathrm{Classic}$ \\ TCM Department, The Affiliated Hospital of \\ Shandong University of TCM, Jinan, China; \\ ${ }^{4}$ Acupuncture and Massage Department, \\ Hangzhou Qihuang Traditional Chinese \\ Medicine Clinic, Hangzhou, China; ${ }^{5}$ TCM \\ and Rehabilitation Department, The Third \\ Hospital of Ulanchap, Ulanchap, China \\ *These authors contributed equally to this \\ work
}

This article was published in the following Dove Press journal: Journal of Pain Research

\begin{abstract}
Background: Acupuncture therapy plays a pivotal role in pain relief, and increasing evidence demonstrates that mast cells (MCs) may mediate acupuncture analgesia. The present study aims to investigate the role of MCs in acupuncture analgesia using c-kit gene mutant-induced MC-deficient rats.
\end{abstract}

Materials and methods: WsRC-Ws/Ws rats and their wild-type (WT) littermates (WsRC-+/+) were used. The number of MCs in skin of ST36 area was compared in two rats after immunofluorescence labeling. Mechanical withdrawal latency (MWL), mechanical withdrawal threshold (MWT), and thermal withdrawal latency (TWL) were measured on bilateral plantar for pain threshold evaluation before and after each stimulus. Acupuncture- and moxibustion-like stimuli $\left(43^{\circ} \mathrm{C}, 46^{\circ} \mathrm{C}\right.$ heat, $1 \mathrm{~mA}$ electroacupuncture [EA], $3 \mathrm{~mA}$ EA, and manual acupuncture [MA]) were applied randomly on different days.

Results: Fewer MCs were observed in the skin of ST36 in mutant rats compared to WT rats $(P<0.001)$. For pain thresholds, MWL and MWT were higher in WsRC-Ws/Ws compared to WsRC-+/+ on bilateral paws $(P<0.05)$, but TWL was not different between the two rats $(P>0.05)$. Bilateral MWL and MWT in WsRC-+/+ rats increased significantly after each stimulus compared to baseline $(P<0.01, P<0.001)$. In $\mathrm{WsRC}-\mathrm{Ws} / \mathrm{Ws}$ rats, only noxious stimuli could produce antinociceptive effects for mechanical pain $\left(46^{\circ} \mathrm{C}, 3 \mathrm{~mA} \mathrm{EA}, \mathrm{MA}\right)(P<0.01, P<0.001)$. Additionally, the net increases in MWL and MWT induced by most stimuli were greater in WT than in mutant rats $(P<0.05)$. For thermal nociception, either high- or low-intensity stimuli could significantly augment TWL in two rats $(P<0.001)$, and the net increases of TWL evoked by most stimuli were to the same extent in two genetic variants.

Conclusion: MCs influence the basic mechanical but not thermal pain threshold. MCs participate in acupuncture analgesia in mechanical but not in thermal nociception, in that MC deficiency may attenuate the mechanical analgesia evoked by high-intensity stimuli and eliminate analgesia provoked by low-intensity stimuli.

Keywords: WsRC-Ws/Ws rats, tryptase, stimulus intensity, mechanical withdrawal threshold, thermal withdrawal latency

\section{Introduction}

Acupuncture therapy has been practiced in China for thousands of years and is used to treat various dysfunctional conditions around the world, ${ }^{1-4}$ particularly for pain relief. Nowadays, the generally accepted mechanisms of acupuncture mainly focus on endogenous opiates and their receptors in the central nervous system, ${ }^{5}$ serotonin and related descending pain inhibitory pathways, ${ }^{6}$ and gate control at the spinal level. ${ }^{7}$ In recent years, several investigations have reported that mast cells (MCs) are involved in acupuncture-induced
Correspondence: Xinyan Gao; Bing Zhu Institute of Acupuncture and Moxibustion, China Academy of Chinese Medical Sciences, 16 Nanxiaojie Street, Dongzhimennei, Beijing 100700, China

Tel +86 I06 4089422

Email gaoxy@mail.cintcm.ac.cn; zhubing@mail. cintcm.ac.cn 
analgesia. ${ }^{8-10}$ MCs are immune cells produced by bone marrow and are ubiquitous throughout the body. ${ }^{11}$ They are mainly distributed in tissues that interact with the external environment, such as the skin, the gastrointestinal tract, the respiratory tract, ${ }^{12}$ the dura mater of the spine, and the meninges of the brain. ${ }^{13,14}$ MCs are activated by inflammation, tissue damage, and physical stimuli, and they release many kinds of bioactive substances via degranulation, such as tryptase, histamines, serotonin, ATP, interleukins 1-6, and many more. ${ }^{9,14,15}$ Dysregulation of $\mathrm{MC}$ activation contributes to a range of abnormal physiologic responses to thermal, mechanical, and ultraviolet stimuli, and also associated with pathologic conditions such as Alzheimer's disease, anxiety, bladder pain syndrome, and nociception. ${ }^{16}$ Previous studies demonstrated that MCs not only participate in peripheral pain transmission, but also play an important role in central integration of pain. ${ }^{14}$ Recently, several lines of evidence have demonstrated that the number of MCs and their degranulation ratio increases significantly following acupuncture, ${ }^{17}$ moxibustion, ${ }^{18}$ and laser acupuncture.${ }^{8}$ Furthermore, ATP released by $\mathrm{MC}$ degranulation serves as a possible mediator for acupuncture analgesia. ${ }^{9}$

However, previous studies mainly observed relationships between MCs and acupuncture analgesia through molecular biology methods, and all subjects were normal rats or mice, failing to elucidate a causal effect of MCs on pain sensation. With the development of genetic engineering, a new mutant rat strain named $\mathrm{WsRC}_{\mathrm{S}} \mathrm{W} / \mathrm{W}$ s has been identified as an ideal animal model to study MCs. ${ }^{19-21}$ WsRc-Ws/Ws rats are found with a 12-base deletion in the tyrosine kinase domain of the $c$-kit gene, ${ }^{22,23}$ which affects MC maturation. ${ }^{11,24}$ These mutant rats have been used to study the role of MCs in visceral hyperalgesia, bladder activity, and stress; ${ }^{20,25,26}$ however, it is yet unknown if MC deficiency influences basal pain sensation and acupuncture-induced analgesia. Therefore, the present study using WsRC-Ws/Ws rats was designed to investigate: 1) the corresponding relationship between MCs and basal pain sensation, and 2) the influence of MC deficiency on the analgesic efficacy induced by acupuncture and moxibustion intervention.

\section{Materials and methods}

\section{Animals}

This study was carried out in strict accordance with the recommendations in the Guide for the Care and Use of Laboratory Animals of the National Institutes of Health. The protocol was approved by the Committee on Ethics of Animal Experiments of Institute of Acupuncture and Moxibustion, China Academy of Chinese Medical Sciences
(Permit Number: 20110510-001), and all efforts were made to minimize animal suffering. Male MC-deficient rats (WsRCWs/Ws, $n=15$ ) and their WT littermates (WsRC-+/+, n=15) were purchased from SLC (Japan SLC, Inc., Tokyo, Japan). Mutant WsRC-Ws/Ws rats have the tyrosine kinase domain of c-kit genes deleted, ${ }^{27} \mathrm{MC}$ deficiency begins at 10 weeks after birth. ${ }^{22,25,28,29}$ Rats were housed in clean animal facilities in which the temperature was maintained at $24^{\circ} \mathrm{C} \pm 0.5^{\circ} \mathrm{C}$, humidity at $60 \%-70 \%$, and noise levels $<60 \mathrm{~dB}$. All animals were housed in groups of two or three with ad libitum access to standard chow and water. The animals were maintained on a standard 12-hour light-dark cycle (dark cycle 8:00 PM-8:00 AM) and were allowed to acclimate to the housing conditions for 7 days prior to the experiment.

\section{Assessment of mechanical withdrawal latency (MWL) and mechanical withdrawal threshold (MWT)}

Before acupuncture and moxibustion stimuli modalities performed, baseline mechanical withdrawal latency (MWL), mechanical withdrawal threshold (MWT), and thermal withdrawal latency (TWL) in the bilateral hind paws were measured in the WsRC-Ws/Ws and WsRC-+/+ rats. Then, noxious $\left(46^{\circ} \mathrm{C}, 3 \mathrm{~mA}\right.$ electroacupuncture [EA], manual acupuncture [MA]) or innocuous $\left(43^{\circ} \mathrm{C}, 1 \mathrm{~mA} \mathrm{EA}\right)$ stimuli mimicking acupuncture and moxibustion were randomly applied on the left hind limb from the second to sixth day with each stimulus performed once per day and at the same time (9:00 AM), because previous studies revealed that $46^{\circ} \mathrm{C}, 3$ mA EA and MA stimuli can activate $\mathrm{A} \delta$ - and C-fibers. ${ }^{30-34} \mathrm{All}$ rats were subjected to the same regime on each day. MWL, WMT, and TWL of the bilateral hind paws were re-evaluated after each stimulus. The Dynamic Plantar Anesthesiometer (37450; UGO Basile, Milan, Italy), an automated von Freytype system, ${ }^{35,36}$ was used to test the MWL and MWT and evaluate mechanical pain threshold before and after each modality. Rats were placed in a transparent partitioned chamber with a stainless-steel mesh bottom and moved freely for $30 \mathrm{~min}$. Mechanical force with a constant rate of $1 \mathrm{~g} / \mathrm{s}$ and cutoff force of $36 \mathrm{~g}$ were exerted by an ascending tip in the middle of the hind paw. The rapid withdrawal latency and withdrawal force were measured. Each paw was tested three times with 15 min interval between each measurement.

In case of the possible influence of daily stimuli on basal mechanical and thermal pain thresholds, we measured basal MWL, MWT, and TWL on the first, third, fourth, and fifth days before acupuncture- or moxibustion-like stimulation. As no statistical differences were found among values of MWL, 
MWT, and TWL in different days, there was no alteration for original response and the rats were still naïve.

\section{Assessment of thermal withdrawal latency (TWL)}

The hind paw withdrawal latency to a noxious thermal stimulus was tested using the plantar test (37370; UGO Basile) ${ }^{35-37}$ Before TWL testing, each rat was placed in a clean plastic compartment over a glass plate and allowed to acclimatize for $30 \mathrm{~min}$. A radiant heat source (infrared intensity: 30 , cutoff time: $15 \mathrm{~s}$ ) beneath the glass plate was placed directly under the plantar of the left or right hind paws, with 15 min interval between two consecutive tests. The latency was recorded when a paw-flick response was elicited. Each rat was tested three times and the average value was collected.

\section{Acupuncture stimulation}

For acupuncture intervention, animals were immobilized without anesthesia in a custom-made fabric sock with holes in it. The influence of restraint stress for rats was also evaluated, and no significant effect was found (see "Results" section). Two acupuncture needles $(0.25 \times 25 \mathrm{~mm}$; Huatuo, Suzhou Medical Co. Ltd., Jiangsu, China) were inserted into left ST36, which is located below the capitulum fibulae and lateral to the tibia, and adjacent non-acupoint at a depth of 1.5-2 $\mathrm{mm}$. EA stimulus with parameters of $20 \mathrm{~Hz}$ and 1 or $3 \mathrm{~mA}$ was generated by HANS-200A Analgesia Apparatus (Nanjing Gensun Medical Technology Co. Ltd., Beijing, China) and was applied to each rat for $10 \mathrm{~min}$. For MA treatment, only one needle was inserted into left ST36 at a depth of 1.5-2 $\mathrm{mm}$, and the needle was twisted clockwise and anticlockwise at $2 \mathrm{~Hz}$ frequency for $10 \mathrm{~min}$. Ten minutes after stimulation, MWL, MWT, and TWL of the rats were re-evaluated.

\section{Moxibustion-like thermal stimulation}

For innocuous warm or noxious heat stimuli, rats were also restrained with the custom-made fabric socks. The left hind paw was stimulated with warm $\left(43^{\circ} \mathrm{C}\right)$ or hot $\left(46^{\circ} \mathrm{C}\right)$ water using a thermostatic water bath for $10 \mathrm{~min}$. Ten minutes after stimulation, MWL, MWT, and TWL were re-evaluated with the same method described earlier.

\section{Immunofluorescent labeling of MCs}

Rats ( $n=5$ per experimental group) were anesthetized by urethane $(1.0 \mathrm{~g} / \mathrm{kg})$, and immediately perfused transcardially with $100 \mathrm{~mL}$ of $0.9 \%$ saline, followed by $300 \mathrm{~mL} 4 \%$ paraformaldehyde in $0.1 \mathrm{M}$ phosphate-buffered saline (PBS,
$\mathrm{pH}$ 7.4). The skin tissue in the area of ST36 $\left(\sim 3 \times 3 \mathrm{~mm}^{2}\right)$ was dissected out from the left hind limb and stored in $25 \%$ sucrose PBS at $4^{\circ} \mathrm{C}$.

The skin was frozen in optimal cutting temperature compound (RSA Group, Kalamazoo, Michigan, USA), and $20 \mu \mathrm{m}$ sections were cut using a cryostat (Thermo, Microm International FSE, Walldorf, Germany). The staining methods were as follows: the sections were washed twice for $10 \mathrm{~min}$ in $0.1 \mathrm{M}$ PBS ( $\mathrm{pH} 7.4$ ), then incubated for $30 \mathrm{~min}$ in blocking solution containing 3\% normal donkey serum and $0.5 \%$ Triton X-100 in PBS. After blocking, the sections were incubated overnight in mouse monoclonal anti-MC tryptase antibody (1:1000; Abcam, Cambridge, UK). On the following day, after washing three times with $0.1 \mathrm{M} \mathrm{PBS}$, the sections were incubated in Alexa Fluor 488 Phalloidin (1:500; Molecular Probes, Eugene, OR, USA) for $2 \mathrm{~h}$ at room temperature followed by three washes with $0.1 \mathrm{M}$ PBS. Then, the sections were coverslipped with $50 \%$ glycerin. Fluorescence was visualized using a light microscope (Eclipse; Nikon Co., Tokyo, Japan).

\section{Statistics}

Data sets with normal distributions were analyzed using the paired $t$-test for differences within groups before and after intervention. Independent $t$-tests were used to compare differences between two groups. One-way analysis of variance (ANOVA) with least significant difference multiple comparison procedure was used to compare differences within each group. Kruskal-Wallis test was used if the data did not satisfy homogeneity of variance. In this study, $P<0.05$ was set as the significance threshold for all comparisons. All data are presented as mean \pm standard error of the mean. The analyses were implemented with SPSS version 20.0 (IBM Corp., Armonk, NY, USA).

\section{Results}

\section{Tryptase expression was downregulated in WsRC-Ws/Ws rats}

To assess the influence of the c-kit gene mutant on $\mathrm{MC}$ expression, the quantity of MCs labeled by tryptase was measured through immunofluorescent staining in WsRC$+/+$ and WsRC-Ws/Ws rats. Normally, tryptase is stored in a fully active form in MC granules, ${ }^{38}$ and this serves as a specific identity marker for MCs. ${ }^{39}$ We found that tryptase expression was significantly reduced in WsRC-Ws/Ws rats as compared to their WT littermates (3.32 \pm 0.89 vs $11.67 \pm 1.06$, independent $t$-test, $P<0.001, \mathrm{n}=5$ ) (Figure 1A-C), similar to results in a previous report. ${ }^{25,29}$ 

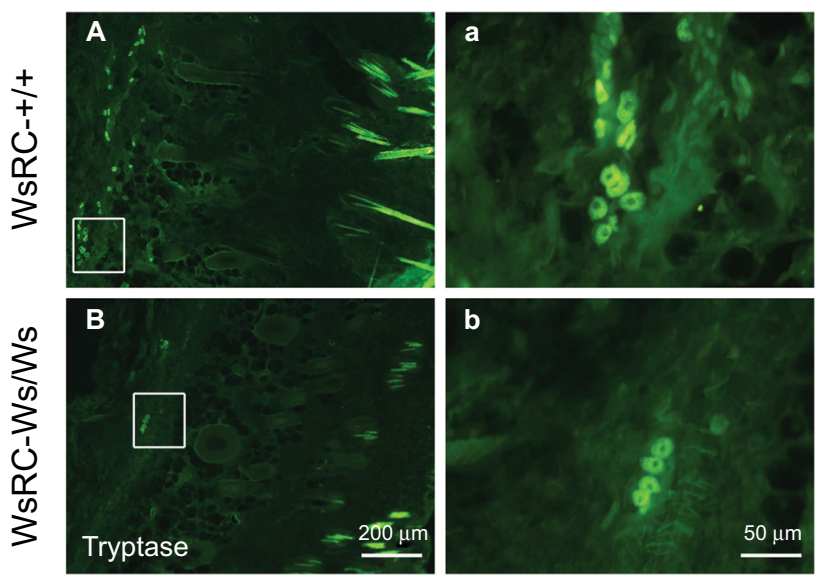

C

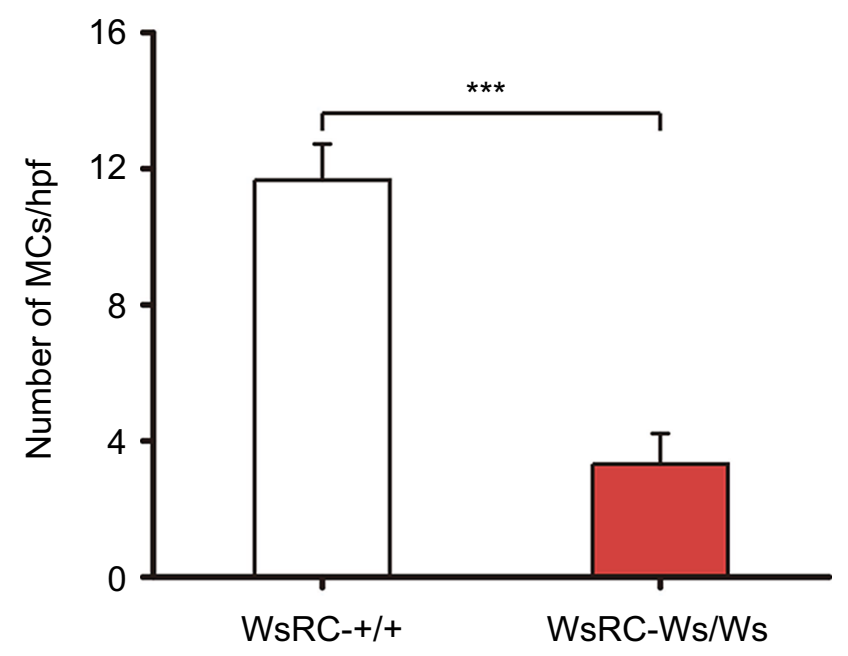

Figure I Immunofluorescence staining for tryptase (MCs) in ST36 skin tissue. Notes: Representative images of $M C$ staining in WsRC-+/+ (A, a) and WsRC-Ws/ Ws $(\mathbf{B}, b)$ rats $(n=5)$. (C) MC count in mutant rats was significantly less than that of WT rats. Data were analyzed using independent $t$-test and expressed as mean \pm standard error of the mean. ${ }^{*} * P<0.00 \mathrm{I}$, vs WsRC-+/+ rats.

Abbreviations: MCs, mast cells; WT, wild-type.

\section{Mechanical but not thermal pain threshold was related to $M C$ expression}

Before testing the influence of MCs on acupuncture analgesia, we first assessed the influence of MCs on pain threshold. To determine whether pain threshold is related to the existence of MCs, we measured the mechanical pain threshold, latency, and thermal pain threshold in WsRC-+/+ and WsRC-Ws/Ws. To control for potential stress effects due to immobilization, paw mechanical and thermal pain thresholds were evaluated. After baseline measurements, rats were restrained in custom-made socks for $10 \mathrm{~min}$. Then, WML, MWT, and TWL were repeatedly recorded. There was no significant change in MWL, MWT, and TWL after restraint as compared to baseline $(P>0.05$, paired $t$-test, $\mathrm{n}=15)$. Mechanical threshold measurements MWL and MWT were greater in WsRC-Ws/ Ws as compared to WsRC-+/+ rats on bilateral paws (MWL: left, $16.71 \pm 1.01 \mathrm{~s}$ vs $13.99 \pm 0.74 \mathrm{~s}$; right, $16.04 \pm 0.71 \mathrm{~s}$ vs $13.70 \pm 0.57 \mathrm{~s}$; MWT: left, $16.94 \pm 1.00 \mathrm{~g}$ vs $14.17 \pm 0.74 \mathrm{~g}$; right, $16.25 \pm 0.7 \mathrm{~g}$ vs $13.89 \pm 0.53 \mathrm{~g}$; independent $t$-test, all $P<0.05$, $\mathrm{n}=15$ ) (Figure 2A and B). However, no significant differences were found between WsRC-Ws/Ws and WsRC-+/+ rats for the thermal pain threshold measurement TWL on bilateral paws (left: $6.06 \pm 0.24 \mathrm{~s}$ vs $5.65 \pm 0.16 \mathrm{~s}$; right, $5.86 \pm 0.23 \mathrm{~s}$ vs $5.61 \pm 0.19 \mathrm{~s}$; independent $t$-test, $P>0.05, \mathrm{n}=15$ ) (Figure $2 \mathrm{C}$ ).

\section{Both the noxious and innocuous stimuli modalities improved mechanical pain threshold in WsRC-+/+ but not WsRC- Ws/Ws rats}

Many previous studies, either preclinical or clinical, have described that acupuncture and moxibustion elevate pain threshold, ${ }^{40-42}$ and the role of MCs in the acupuncture analgesia has been confirmed. ${ }^{9,10,43-46}$ Here, we determined baseline threshold before a noxious $\left(46^{\circ} \mathrm{C}, 3 \mathrm{~mA}\right.$ EA, MA) or innocuous $\left(43^{\circ} \mathrm{C}, 1 \mathrm{~mA} \mathrm{EA}\right)$ stimulus mimicking acupuncture and moxibustion was performed, ${ }^{30-32}$ and then measured analgesic efficacy when each stimulus modality was conducted at different days randomly in WsRC-+/+ and WsRC-Ws/Ws rats.

As shown in Figure 3, bilateral MWL and MWT in WsRC+/+ rats increased significantly after each stimulus, noxious or innocuous, compared to the baseline response latency and threshold before each stimulus. In detail, MWL of the ipsilateral paw was $43^{\circ} \mathrm{C}, 18.61 \pm 1.27 \mathrm{~s} ; 46^{\circ} \mathrm{C}, 19.09 \pm 1.12 \mathrm{~s}$; $1 \mathrm{~mA}, 20.15 \pm 1.17 \mathrm{~s} ; 3 \mathrm{~mA}, 20.56 \pm 1.09 \mathrm{~s} ; \mathrm{MA}, 23.48 \pm 1.18 \mathrm{~s}$ after stimulus vs baseline $13.99 \pm 0.74 \mathrm{~s}$ (one-way ANOVA, $P<0.01$ or $P<0.001, \mathrm{n}=15$, Figure $3 \mathrm{~A}$ ). MWL of the contralateral paw was $43^{\circ} \mathrm{C}, 17.65 \pm 1.12 \mathrm{~s} ; 46^{\circ} \mathrm{C}, 19.96 \pm 0.86 \mathrm{~s}$; $1 \mathrm{~mA}, 19.36 \pm 0.98 \mathrm{~s} ; 3 \mathrm{~mA}, 24.95 \pm 0.86 \mathrm{~s} ; \mathrm{MA}, 23.24 \pm 1.34 \mathrm{~s}$, respectively, after stimulus compared to baseline $13.70 \pm 0.57 \mathrm{~s}$ $(P<0.01$ or $P<0.001$, Figure 3B). Similarly, MWT of the ipsilateral paw was as $43^{\circ} \mathrm{C}, 18.76 \pm 1.24 \mathrm{~g} ; 46^{\circ} \mathrm{C}, 19.29 \pm 1.11 \mathrm{~g}$; $1 \mathrm{~mA}, 19.83 \pm 1.06 \mathrm{~g} ; 3 \mathrm{~mA}, 20.69 \pm 1.08 \mathrm{~g}$; MA, $22.6 \pm 1.23 \mathrm{~g}$ after stimulus compared to baseline $14.17 \pm 0.74 \mathrm{~g}(P<0.01$ or $P<0.001$, Figure $3 \mathrm{C}$ ). MWT of the contralateral paw was $43^{\circ} \mathrm{C}, 17.76 \pm 1.09 \mathrm{~g} ; 46^{\circ} \mathrm{C}, 20.12 \pm 0.84 \mathrm{~g} ; 1 \mathrm{~mA}, 19.72 \pm 0.92 \mathrm{~g}$; $3 \mathrm{~mA}, 25.11 \pm 0.88 \mathrm{~g}$; MA, $22.74 \pm 1.5 \mathrm{~g}$ after stimulus vs baseline $13.89 \pm 0.53 \mathrm{~g}(P<0.01$ or $P<0.001$, Figure 3D).

Comparatively, in WsRC-Ws/Ws rats, only high-intensity or noxious stimulus $\left(46^{\circ} \mathrm{C}, 3 \mathrm{~mA} \mathrm{EA}, \mathrm{MA}\right)$ induced a significant enhancement of mechanical response latency MWL and threshold MWT bilaterally. Low-intensity stimuli $\left(43^{\circ} \mathrm{C}\right.$ 
A
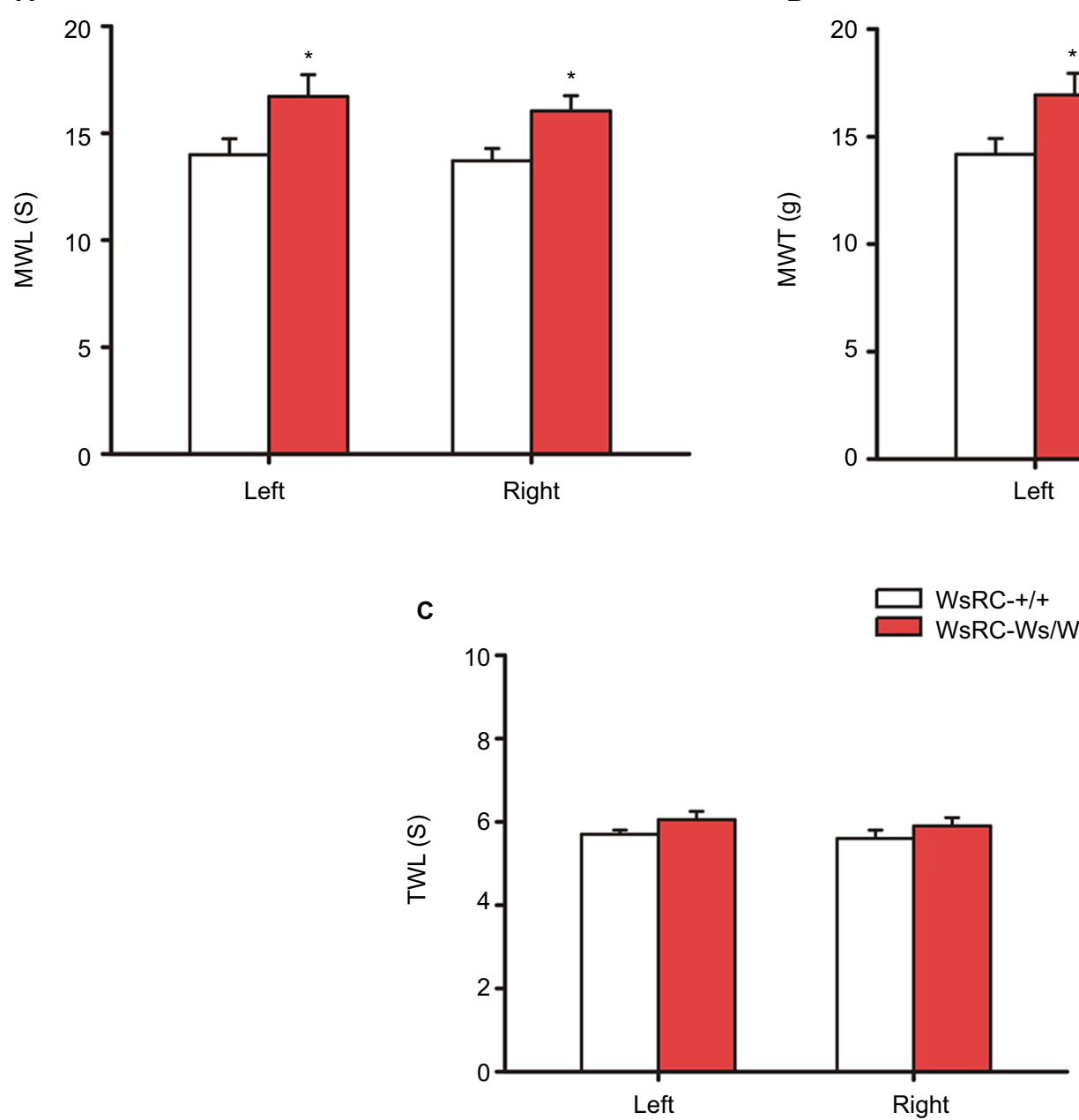

B

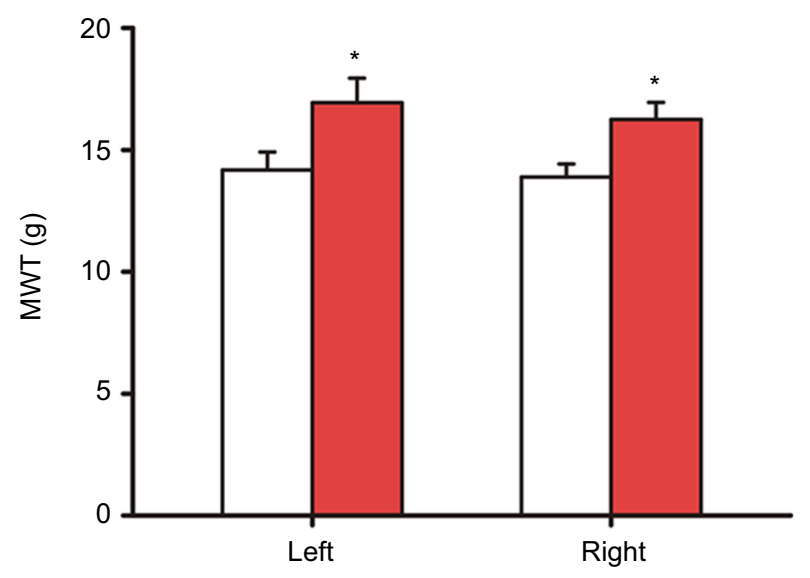

Figure 2 Baseline MWL, MWT, and TWL in left and right hind paws in WsRC-+/+ and WsRC-Ws/Ws rats.

Notes: Baseline MWL (A) and MWT (B) in WT and mutant rats $(n=15)$. (C) Baseline TWL in two rats. Data were analyzed using independent $t$-test and expressed as mean \pm standard error of the mean. $* P<0.05$, compared with WsRC-+/+ rats. WsRC-Ws/Ws rats showed a longer MWL and higher MWT ( $P<0.05)$ than $W T$ rats.

Abbreviations: MWL, mechanical withdrawal latency; MWT, mechanical withdrawal threshold; TWL, thermal withdrawal latency; WT, wild-type.

and $1 \mathrm{mAEA}$ ) did not have apparent efficacy. Data are shown in detail in Figure 3. MWL of the ipsilateral paw was $46^{\circ} \mathrm{C}$, $19.97 \pm 1.15 \mathrm{~s} ; 3 \mathrm{~mA}, 19.94 \pm 1.03 \mathrm{~s} ; \mathrm{MA}, 21.57 \pm 1.74 \mathrm{~s}$ after stimulus vs baseline $13.99 \pm 0.74 \mathrm{~s}$ (one-way ANOVA, $P<0.05$, $\mathrm{n}=15$, Figure $3 \mathrm{~A}$ ). MWL of the contralateral paw was $46^{\circ} \mathrm{C}$, $19.97 \pm 1.16 \mathrm{~s} ; 3 \mathrm{~mA}, 20.46 \pm 1.35 \mathrm{~s} ;$ MA, $21.60 \pm 1.94 \mathrm{~s}$, respectively, after stimulus as compared to baseline $13.70 \pm 0.57 \mathrm{~s}$ $\left(P<0.01\right.$, Figure 3B). MWT of the ipsilateral paw was $46^{\circ} \mathrm{C}$, $19.97 \pm 1.15 \mathrm{~g} ; 3 \mathrm{~mA}, 20.11 \pm 1.02 \mathrm{~g}$; MA, $21.72 \pm 1.72 \mathrm{~g}$ after stimulus as compared to baseline $16.94 \pm 1.00 \mathrm{~g}(P<0.05$, Figure $3 \mathrm{C})$. MWT of the contralateral paw was $46^{\circ} \mathrm{C}$, $20.1 \pm 1.14 \mathrm{~g} ; 3 \mathrm{~mA}, 20.66 \pm 1.33 \mathrm{~g}$; MA, $21.95 \pm 2.1 \mathrm{~g}$ after stimulus vs baseline $16.25 \pm 0.70 \mathrm{~g}(P<0.01$ or $P<0.001$, Figure 3D). For low-intensity interventions $\left(43^{\circ} \mathrm{C}\right.$ and $\left.1 \mathrm{~mA} \mathrm{EA}\right)$, no significant changes were induced on MWL of ipsilateral $\left(43^{\circ} \mathrm{C}, 18.11 \pm 0.73 \mathrm{~s} ; 1 \mathrm{~mA}, 19.8 \pm 1.16 \mathrm{~s}\right.$; compared to prestimulus $16.71 \pm 1.01 \mathrm{~s}, P>0.05$, Figure $3 \mathrm{~A}$ ) and contralateral $\left(43^{\circ} \mathrm{C}, 18.25 \pm 0.69 \mathrm{~s} ; 1 \mathrm{~mA}, 19.97 \pm 1.16 \mathrm{~s}\right.$; compared to prestimulus $16.04 \pm 0.71 \mathrm{~s}, P>0.05$, Figure $3 \mathrm{~B}$ ), or on MWT of ipsilateral $\left(43^{\circ} \mathrm{C}, 18.45 \pm 0.73 \mathrm{~g} ; 1 \mathrm{~mA}, 19.97 \pm 1.15 \mathrm{~g}\right.$; compared to prestimulus $16.94 \pm 1.00 \mathrm{~g}, P>0.05$, Figure $3 \mathrm{C}$ ) and contralateral $\left(43^{\circ} \mathrm{C}, 18.48 \pm 0.67 \mathrm{~g} ; 1 \mathrm{~mA}, 20.1 \pm 1.14 \mathrm{~g}\right.$; compared to prestimulus $16.25 \pm 0.70 \mathrm{~g}, P>0.05$, Figure 3D).

These results demonstrate that MC deficiency in WsRCWs/Ws rats in our study abolishes analgesic effects induced by acupuncture- and moxibustion-like stimuli with innocuous intensities, whereas the analgesic effects of noxious stimuli are preserved. This result indicates that MCs participate in acupuncture or moxibustion analgesia, depending on the intervention intensity. 

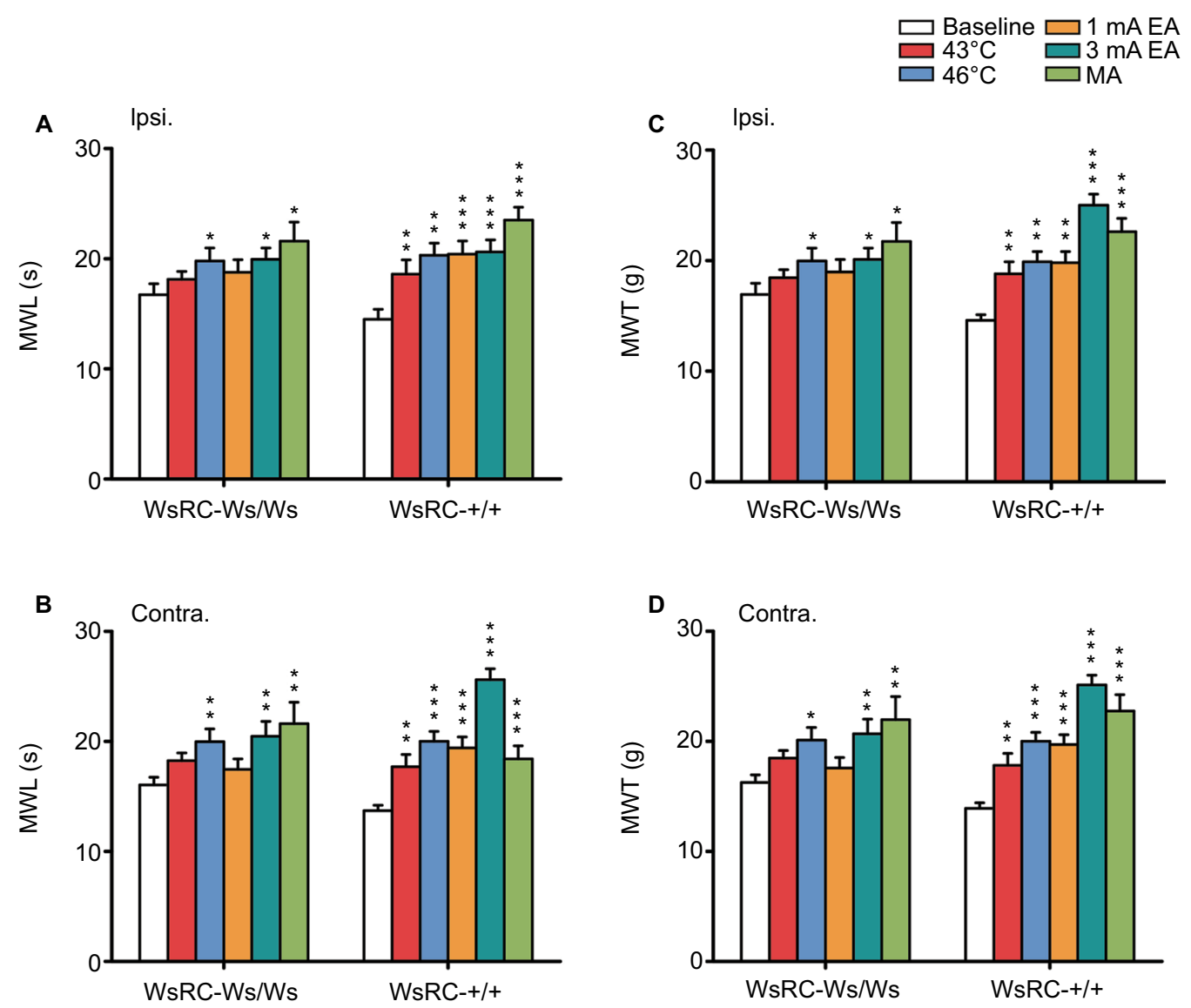

Figure $3 \mathrm{MC}$ deficiency blocked the effect of low-intensity stimuli on mechanical pain sensation.

Notes: Baseline MWL and MWT data after different stimuli at $43^{\circ} \mathrm{C}$ and $46^{\circ} \mathrm{C}$ heat, $1 \mathrm{~mA}$ and $3 \mathrm{~mA} E A$, and MA were collected in plantar test. Ipsilateral (A) and contralateral (B) paw MWL were increased by acupuncture- and moxibustion-like stimulation, either low or high intensities in WT rats, whereas only high intensity of stimulus increased ipsilateral and contralateral paw MWL in mutant rats. Ipsilateral (C) and contralateral (D) paw MWT were increased by acupuncture- and moxibustionlike stimulation, either low or high intensities in WT rats, whereas only high intensity of stimulus increased ipsilateral and contralateral paw MWL and MWT in mutant rats. Data were analyzed using one-way analysis of variance and least significant difference for post hoc analysis. $* P<0.05$, $* * P<0.0$ I, $* * * P<0.00$ I, compared with baseline $M W L$ or MWT in each group. Data represents the mean \pm standard error of the mean.

Abbreviations: Contra., contralateral; EA, electroacupuncture; Ipsi., ipsilateral; MA, manual acupuncture; MC, mast cell; MWL, mechanical withdrawal latency; MWT, mechanical withdrawal threshold; WT, wild-type.

\section{Mechanical pain threshold was promoted more apparently by different stimulus modalities in WsRC-+/+ rats than in MC-deficient WsRC-Ws/Ws rats}

Next, we investigated whether there were any differences in the range of mechanical threshold elevation between the two genotypes, especially for high-intensity stimuli. In the present study, the elevation range of mechanical threshold was obtained by the value of MWL or MWT after each stimulus minus the value of baseline. Because values of MWL or MWT after each stimulus were higher than baseline with (for stimuli of all intensities in WsRC-+/+ rats and high-intensity stimuli in WsRC-Ws/Ws rats) or without (for low intensities in WsRC-Ws/Ws rats) significant differences (Figure 3), difference values obtained were all positive numbers (Figure 4).
Results for the elevation range of mechanical pain threshold in bilateral MWL and MWT are shown in Figure 4. After high-intensity or noxious stimuli (which induced a significant elevation of mechanical pain threshold in both rats), the enhancement ranges of MWL on bilateral paws in WsRC-+/+ rats were greater than those in WsRC-Ws/Ws rats (ipsilateral: $46^{\circ} \mathrm{C}, 5.66 \pm 1.16 \mathrm{~s}$ vs $1.23 \pm 1.72 \mathrm{~s} ; 3 \mathrm{~mA}$, $7.04 \pm 1.34 \mathrm{~s}$ vs $2.13 \pm 1.55 \mathrm{~s} ;$ MA, $9.0 \pm 1.34 \mathrm{~s}$ vs $4.86 \pm 1.33 \mathrm{~s}$; independent $t$-test, $P<0.05, \mathrm{n}=15$, Figure $4 \mathrm{~A}$; contralateral: $46^{\circ} \mathrm{C}, 6.3 \pm 0.98 \mathrm{~s}$ vs $2.56 \pm 1.37 \mathrm{~s} ; 3 \mathrm{~mA}, 11.28 \pm 1.10 \mathrm{~s}$ vs $4.43 \pm 1.53 \mathrm{~s} ; \mathrm{MA}, 9.57 \pm 1.48 \mathrm{~s}$ vs $4.57 \pm 1.10 \mathrm{~s} ; P<0.05$ or $P<0.01$, Figure 4B). Similarly, the MWT elevation changes induced by noxious stimuli on bilateral paws in $\mathrm{WsRC}_{\mathrm{s}}+\mathrm{+}+$ rats were greater than those in $\mathrm{WsRC}_{\mathrm{s}} \mathrm{Ws}_{\mathrm{s}} / \mathrm{Ws}_{\mathrm{s}}$ rats (ipsilateral: $46^{\circ} \mathrm{C}, 5.68 \pm 1.15 \mathrm{~g}$ vs $1.21 \pm 1.69 \mathrm{~g} ; 3 \mathrm{~mA}, 6.97 \pm 1.34 \mathrm{~g}$ vs $2.08 \pm 1.53 \mathrm{~g}$; MA, $8.96 \pm 1.13 \mathrm{~g}$ vs $4.78 \pm 1.01 \mathrm{~g} ; P<0.05$, 
A

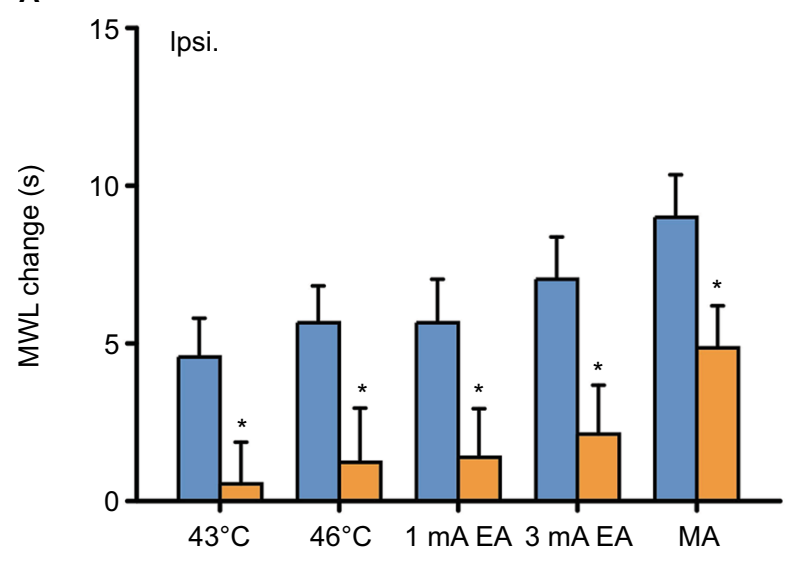

B

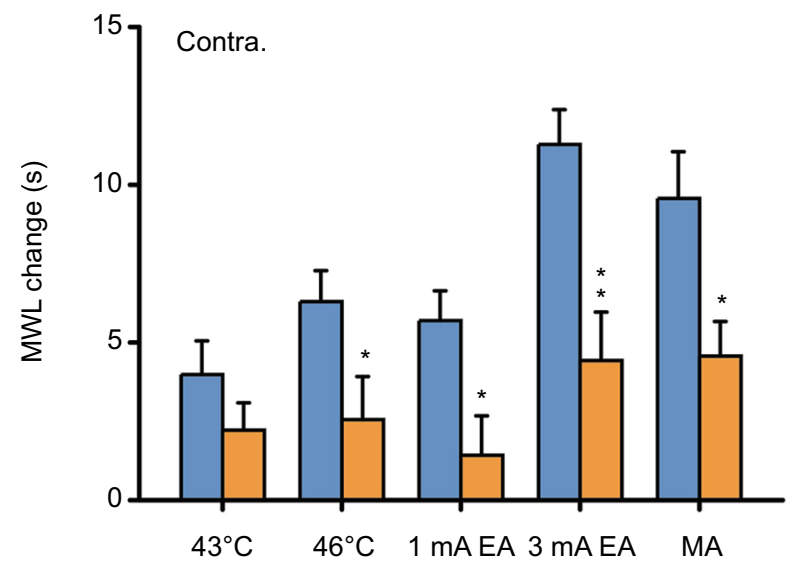

C

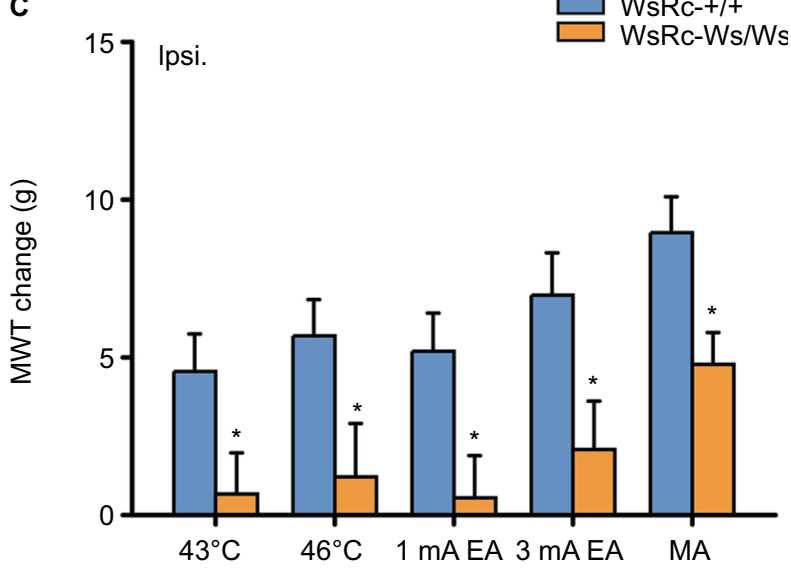

D

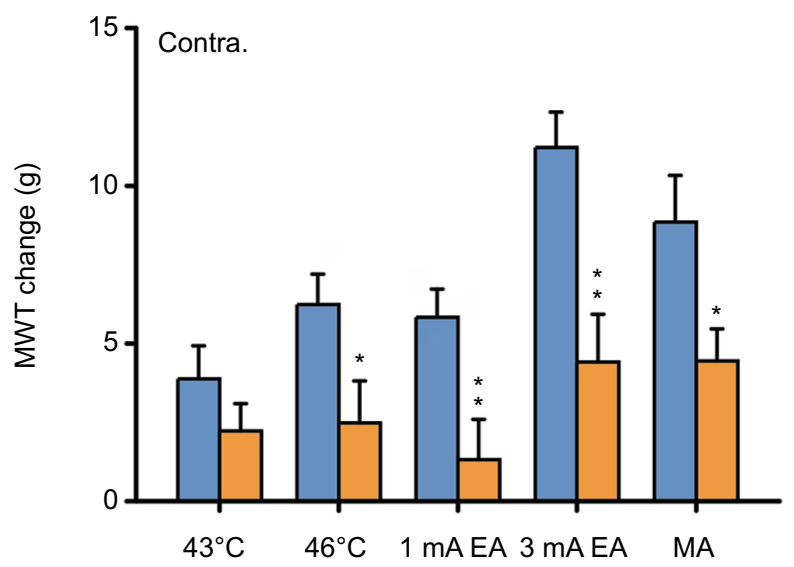

Figure $4 \mathrm{MC}$ deficiency impaired the effect of acupuncture and moxibustion-like stimulation on the improvement of MWL and MWT.

Notes: Data are represented as net increases of MWL and MWT (subtracting the baseline value from poststimulus values). Net increases of MWL in ipsilateral (A) and contralateral (B) paws in WsRC-+l+ and WsRC-Ws/Ws rats $(n=15)$. Net increases of MWT in ipsilateral (C) and contralateral (D) paws in WsRC-+l+ and WsRC-Ws/Ws rats $(n=15)$. Independent $t$-tests, ${ }^{*} P<0.05,{ }^{*} * P<0.01$, vs $W s R C-+/+$ rats. $M C$ deficiency significantly decreased most effects of acupuncture and moxibustion-like stimuli on MWL and MWT in WsRC-Ws/Ws rats as compared to those in WsRC-+/+ rats. Data are represented as mean \pm standard error of the mean.

Abbreviations: Contra., contralateral; Ipsi., ipsilateral; MC, mast cell; MWL, mechanical withdrawal latency; MWT, mechanical withdrawal threshold; EA, electroacupuncture; MA, manual acupuncture..

Figure 4C; contralateral: $46^{\circ} \mathrm{C}, 6.23 \pm 0.97 \mathrm{~g}$ vs $2.48 \pm 1.33 \mathrm{~g}$; $3 \mathrm{~mA}, 11.22 \pm 1.12 \mathrm{~g}$ vs $4.42 \pm 1.51 \mathrm{~g} ; \mathrm{MA}, 8.85 \pm 1.48 \mathrm{~g}$ vs $4.45 \pm 1.01 \mathrm{~g} ; P<0.05$ or $P<0.01$, Figure 4D).

In contrast, after low-intensity or innocuous stimuli, which induced significant elevation on mechanical threshold only in WsRC-+/+ rats (Figure 3), the increased ranges of MWL on bilateral paws in WsRC-+/+ rats were greater than those in WsRC-Ws/Ws rats, except for the $43^{\circ} \mathrm{C}$ stimulus on the contralateral paw (ipsilateral: $43^{\circ} \mathrm{C}, 4.57 \pm 1.23 \mathrm{~s}$ vs $0.54 \pm 1.33 \mathrm{~s} ; 1 \mathrm{~mA}, 5.66 \pm 1.37 \mathrm{~s}$ vs $1.39 \pm 1.54 \mathrm{~s}$; both $P<0.05$, independent $t$-test, $\mathrm{n}=15$, Figure $4 \mathrm{~A}$; contralateral: $43^{\circ} \mathrm{C}$, $3.98 \pm 1.07 \mathrm{~s}$ vs $2.22 \pm 0.87 \mathrm{~s}, P>0.05 ; 1 \mathrm{~mA}, 5.69 \pm 0.95 \mathrm{~s}$ vs $1.42 \pm 1.25 \mathrm{~s} ; P<0.05$, Figure 4B). The elevation ranges of MWT on bilateral paws in WsRC-+/+ rats were greater than those in WsRC-Ws/Ws rats, except for the $43^{\circ} \mathrm{C}$ stimulus on the contralateral paw (ipsilateral: $43^{\circ} \mathrm{C}, 4.55 \pm 1.19 \mathrm{~g}$ vs $0.67 \pm 1.3 \mathrm{~g} ; 1 \mathrm{~mA}, 5.19 \pm 1.22 \mathrm{~g}$ vs $0.54 \pm 1.34 \mathrm{~g}$; both $P<0.05$, Figure $4 \mathrm{C}$; contralateral: $43^{\circ} \mathrm{C}, 3.88 \pm 1.05 \mathrm{~g}$ vs $2.23 \pm 0.86 \mathrm{~g}, P>0.05 ; 1 \mathrm{~mA}, 5.84 \pm 0.89 \mathrm{~g}$ vs $1.32 \pm 1.28 \mathrm{~g}$; $P<0.05$, Figure 4D).

\section{MC deficiency did not alter the effect of different stimulus modalities on thermal pain threshold}

To further explore the role of MCs on acupuncture analgesia for thermal nociception, TWL was examined before and after each stimulus. Our data demonstrate quite different but interesting implications that both noxious and innocuous stimuli elevated thermal pain threshold in both WsRC-+/+ and WsRC-Ws/Ws rats (Figure 5).

For WsRC- $+/+$ rats, TWL of the ipsilateral paw was $43^{\circ} \mathrm{C}$, $7.26 \pm 0.33 \mathrm{~s} ; 46^{\circ} \mathrm{C}, 10.72 \pm 0.28 \mathrm{~s} ; 1 \mathrm{~mA}, 10.47 \pm 0.37 \mathrm{~s} ; 3 \mathrm{~mA}$, 
$10.98 \pm 0.23 \mathrm{~s} ; \mathrm{MA}, 10.12 \pm 0.54 \mathrm{~s}$ after stimulus vs baseline $5.65 \pm 0.16 \mathrm{~s}$ (one-way ANOVA, $P<0.001, \mathrm{n}=15$, Figure $5 \mathrm{~A}$ ). TWL of the contralateral paw was $43^{\circ} \mathrm{C}, 6.84 \pm 0.30 \mathrm{~s} ; 46^{\circ} \mathrm{C}$, $10.92 \pm 0.30 \mathrm{~s} ; 1 \mathrm{~mA}, 10.32 \pm 0.36 \mathrm{~s} ; 3 \mathrm{~mA}, 11.26 \pm 0.4 \mathrm{~s}$; MA, 9.63 $\pm 0.42 \mathrm{~s}$ after stimulus vs baseline $5.61 \pm 0.19 \mathrm{~s}$ $(P<0.001$, Figure 5B). Data obtained from WsRC-Ws/Ws rats were quite similar. TWL of the ipsilateral paw was $43^{\circ} \mathrm{C}$, $7.83 \pm 0.29 \mathrm{~s} ; 46^{\circ} \mathrm{C}, 10.46 \pm 0.36 \mathrm{~s} ; 1 \mathrm{~mA}, 11.13 \pm 0.46 \mathrm{~s} ; 3 \mathrm{~mA}$, $10.29 \pm 0.23 \mathrm{~s} ; \mathrm{MA}, 9.33 \pm 0.28 \mathrm{~s}$ after stimulus vs baseline $6.06 \pm 0.24 \mathrm{~s}(P<0.001$, Figure $5 \mathrm{~A})$. TWL of the contralateral paw was $43^{\circ} \mathrm{C}, 7.53 \pm 0.35 \mathrm{~s} ; 46^{\circ} \mathrm{C}, 10.92 \pm 0.26 \mathrm{~s} ; 1 \mathrm{~mA}$, $10.9 \pm 0.48 \mathrm{~s} ; 3 \mathrm{~mA}, 10.56 \pm 0.21 \mathrm{~s} ; \mathrm{MA}, 9.25 \pm 0.31 \mathrm{~s}$ after stimulus vs baseline $5.86 \pm 0.23 \mathrm{~s}(P<0.001$, Figure 5B).

\section{Thermal pain threshold was enhanced within the same range between WsRC-+/+ and WsRC-Ws/Ws rats}

To determine whether the changes in thermal pain threshold evoked by these stimuli modalities differed between the two
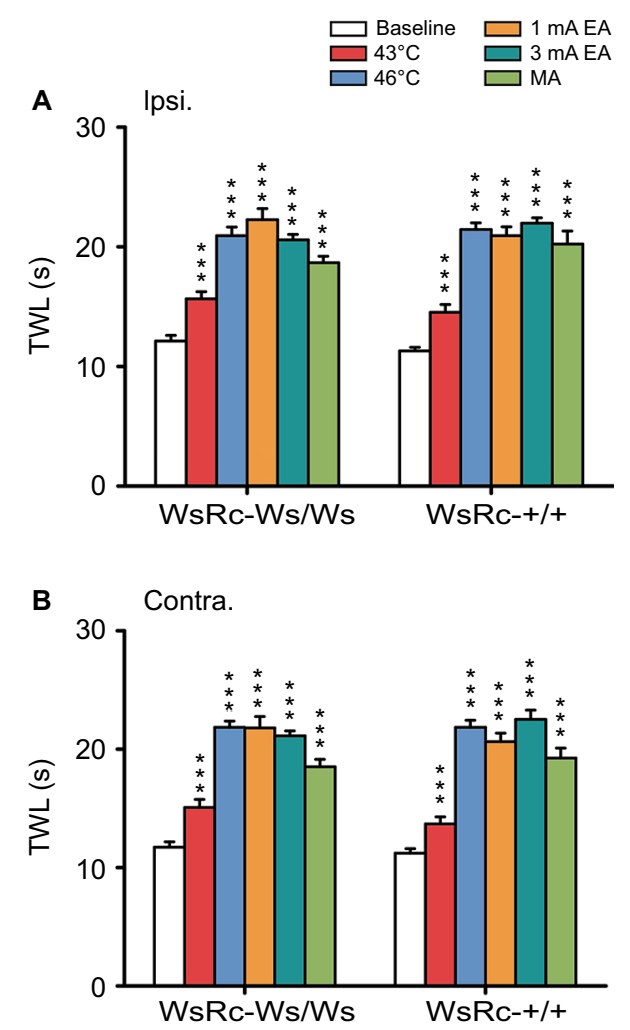

Figure $5 \mathrm{MC}$ deficiency had no effect on TWL improvement induced by acupuncture and moxibustion stimuli.

Notes: Ipsilateral (A) and contralateral (B) hind paw TWL of WsRC-+/+ and WsRC-Ws/Ws rats were increased by different intensities of acupuncture and moxibustion stimuli $(n=15)$. All data were analyzed using one-way analysis of variance and least significant difference for post hoc analysis. $* * * P<0.001$, compared with baseline TWL. Data are expressed as mean \pm standard error of the mean.

Abbreviations: Contra., contralateral; Ipsi., ipsilateral; TWL, thermal withdrawal latency; EA, electroacupuncture; MA, manual acupuncture; MC, mast cell. genotypes, we compared the net elevations of TWL between the two groups. The elevation range of thermal pain threshold was calculated by subtracting the baseline TWL value from each poststimulus TWL value. Because TWL values after each stimulus were greater than baseline with significant differences in each stimulus (at both high and low intensities), differences acquired were all positive values (Figure 6).

After acupuncture- or moxibustion-like stimuli, the magnitude of TWL increase on bilateral paws was the same between WsRC-+/+ and WsRC-Ws/Ws rats, except for the $3 \mathrm{~mA}$ EA stimulus (ipsilateral: $43^{\circ} \mathrm{C}, 1.62 \pm 0.41 \mathrm{~s}$ vs $1.77 \pm 0.23 \mathrm{~s} ; 46^{\circ} \mathrm{C}, 5.08 \pm 0.35 \mathrm{~s}$ vs $4.41 \pm 0.3 \mathrm{~s} ; 1 \mathrm{~mA}$, $4.82 \pm 0.41 \mathrm{~s}$ vs $5.07 \pm 0.49 \mathrm{~s} ;$ MA, $2.23 \pm 0.36 \mathrm{~s}$ vs $2.2 \pm 0.48 \mathrm{~s}$; all $P>0.05 ; 3 \mathrm{~mA}, 5.34 \pm 0.25 \mathrm{~s}$ vs $4.23 \pm 0.28 \mathrm{~s}, P<0.05$, independent $t$-test, $\mathrm{n}=15$, Figure $6 \mathrm{~A}$; contralateral: $43^{\circ} \mathrm{C}$, $1.24 \pm 0.37 \mathrm{~s}$ vs $1.68 \pm 0.33 \mathrm{~s} ; 46^{\circ} \mathrm{C}, 5.31 \pm 0.39 \mathrm{~s}$ vs $5.06 \pm 0.23 \mathrm{~s}$;
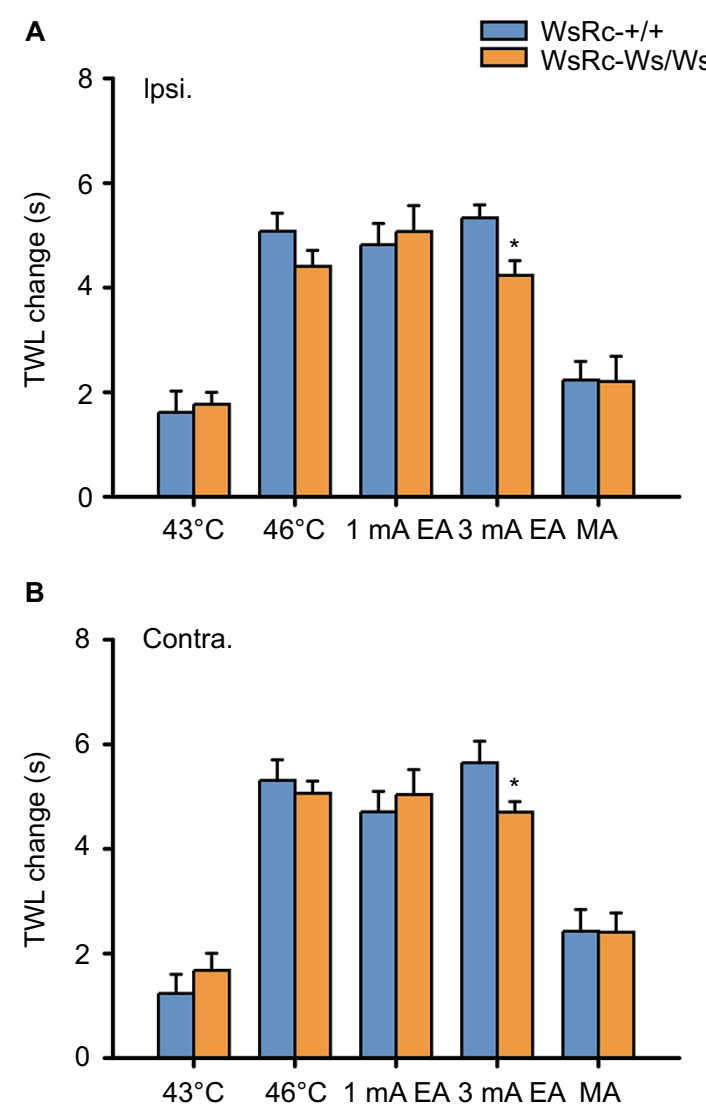

Figure $6 \mathrm{MC}$ deficiency did not influence the effect of acupuncture and moxibustion on TWL increase.

Notes: Data are represented as net increase of TWL (subtracting the baseline value from poststimulus values). Net increase of TWL in ipsilateral $(\mathbf{A})$ and contralateral (B) paws in WsRC-+/+ and WsRC-Ws/Ws rats $(n=15)$. All data were analyzed using independent $t$-test and expressed as mean \pm standard error of the mean. $* P<0.05$, compared with WsRC-+/+ rats. All improvements of TWL were similar in WT and mutant rats except the effect of $3 \mathrm{~mA} \mathrm{EA}$, that the increase of TWL in WT rats was significantly greater than that in mutant rats $(P<0.05)$.

Abbreviations: Contra., contralateral; EA, electroacupuncture; Ipsi., ipsilateral; TWL, thermal withdrawal latency; WT, wild-type; MA, manual acupuncture; MC, mast cell. 
$1 \mathrm{~mA}, 4.71 \pm 0.39 \mathrm{~s}$ vs $5.04 \pm 0.47 \mathrm{~s} ; \mathrm{MA}, 2.42 \pm 0.42 \mathrm{~s}$ vs

$2.41 \pm 0.37 \mathrm{~s}$; all $P>0.05 ; 3 \mathrm{~mA}, 5.65 \pm 0.41 \mathrm{~s}$ vs $4.7 \pm 0.2 \mathrm{~s}$, $P<0.05 ; \mathrm{n}=15$, Figure 5B).

\section{Discussion}

To our knowledge, this is the first preclinical study to investigate the role of MCs in pain sensation and in acupuncture-moxibustion analgesic efficacy using a c-kit gene mutant and MC-ablated rats. In the present study, we measured basic mechanical and thermal pain thresholds and pain threshold changes produced by different stimuli mimicking acupuncture and moxibustion in WT WsRC$+/+$ and c-kit gene mutant WsRC-Ws/Ws rats. First, our results demonstrate that MCs influence mechanical but not thermal pain perception (Figure 2). Secondly, acupunctureand moxibustion-like stimulation elevated mechanical pain threshold in an intensity-dependent manner in WsRC-Ws/Ws but not in WsRC-+/+ rats (Figure 3), and mechanical pain threshold changes were greater in WsRC-+/+ than those in WsRC-Ws/Ws rats (Figure 4), indicating that MCs play a role in acupuncture- and moxibustion-induced analgesic effects for mechanical pain. In contrast, different stimulus modalities elevated thermal pain threshold in both WsRC-+/+ and WsRC-Ws/Ws rats (Figure 5), almost without differences in magnitude (Figure 6). The study implies that MCs influence mechanical pain perception as well as acupuncture-moxibustion analgesia, but do not affect perception and analgesia of thermal pain.

\section{The influence of MCs on nociception}

As immune-serving MCs reside in all tissues ${ }^{11}$ such as skin, mucous membranes, respiratory and gastrointestinal tracts, they are involved in immediate physiologic reactions ("first responders" to external pathogen and allergen exposure) and various pathologic conditions such as visceral diseases, autoimmune disorders, cancer, ischemia-reperfusion injury, anxiety, and Alzheimer's disease. ${ }^{16}$ In the present study, WsRC-Ws/Ws rats were characterized by MC deficiency via a 12-base deletion in the tyrosine kinase domain of the c-kit gene ${ }^{22,23}$ which not only plays a vital role in MC differentiation and function, ${ }^{13}$ but also regulates melanocytes, erythrocyte, and germ cells. ${ }^{47,48}$ Although c-kit mutant mice was the earliest MC-deficient animals and wildly used in experimental work, ${ }^{49-51}$ previous studies demonstrated that the magnitude of MC deficiency was comparable between c-kit mutant mice and c-kit mutant rats. ${ }^{22,27}$ Thus, these mutant rats have been recommended as a viable animal model for investigating the functions of MCs. ${ }^{19-22,27}$ Further, our results also showed that the WsRC-Ws/Ws rats displayed a reduced quantity of $\mathrm{MC}$ distribution in the skin located at the acupoint ST36 (Figure 1).

Previous reports have discussed the underlying mechanisms relating MCs and pain in the central and peripheral nervous systems. ${ }^{9,14,15,45}$ MCs, activated by various nociceptive or inflammatory stimuli, could release tryptase, ATP, histamines, and other various bioactive agents and inflammatory mediators via degranulation, ${ }^{52,53}$ which may act on nociceptors and modulate synaptic transmission and nociception at spinal dorsal horn. ${ }^{14}$ In turn, these agents stimulate nociceptive receptors on sensory endings to release neuromodulators such as substance $\mathrm{P}$, calcitonin gene-related peptide (CGRP), and vasoactive intestinal protein, and activate additional downstream degranulation cascade reactions in MCs. ${ }^{14,54,55}$ Enhanced MC activation and degranulation lead to release of additional algogenic molecules, which arouses nociceptors sensitization and generates perception of pain in the body. ${ }^{56}$

It has been reported that tryptase, a mediator released by activated MCs, could activate protease-activated receptor 2 (PAR-2) and transient receptor potential A1, and then induce mechanical hypersensitivity of vagal C-fibers. ${ }^{57} \mathrm{In}$ addition, PAR- 2 activation also could facilitate the release of nociceptive neuromodulators, such as substance $\mathrm{P}$ and CGRP, in peripheral tissues. ${ }^{58}$ In the present experiments, mutant rats were not so sensitive to mechanical pain stimuli as WT rats, demonstrated by increases in MWL and MWT (Figure 2A and B). This was consistent with our hypothesis that nociceptive fibers were less sensitive for short of active compounds released by MCs degranulation in WsRC-Ws/ Ws rats compared to WsRC-+/+ rats, and WsRC-Ws/Ws rats were dull to mechanical detection. However, an interesting finding in our study was that the basic thermal pain threshold was not influenced by MC deficiency, because TWL was not different between WsRC-Ws/Ws and WsRC-+/+ rats (Figure 2C). Previous reports confirmed that TRPV receptors, related to nociception and may be activated by capsaicin, played a critical role in noxious or innocuous thermal perception. ${ }^{59,60}$ Although relationships between MCs and TRPV receptors under the condition of visceral hyperalgesia were reported, ${ }^{26,42}$ less evidence was supported for somatosensory paresthesia.

In addition, 12-base deletion of the c-kit genomic DNA also leads to fewer number of melanocytes and erythrocyte in skin in WsRC-Ws/Ws rats compared with that in WT rats, but not germ cell. ${ }^{22}$ One study reported that melanocortin-1 receptor (MC1R) expressed in melanocytes played 
a female-specific role in $\kappa$-opioid analgesia by using Mclre mutant mice and correspondingly in humans with multiple MC1R variants. ${ }^{61}$ Similarly, another study of the same group demonstrated a female-specific role of MC1R in acute thermal noxious response and inflammation. ${ }^{62}$ Our experiment was accomplished in male c-kit mutant rats, and the relationship between melanocytes and pain sensation and acupuncture analgesia is still lacking evidences and needs further investigation. In addition, we have failed to find a correlation among the role of erythrocyte, germ cells, pain and acupuncture analgesia.

\section{The role of MCs in acupuncture- and moxibustion-like stimulation analgesia}

Although several lines of evidence have revealed that MCs participate in the mechanism of analgesia produced by acupuncture, ${ }^{10,45}$ moxibustion, ${ }^{18}$ and laser needling, ${ }^{8}$ these studies mainly verified possible biologic mechanisms via pharmaceutical tools such as the MC stabilizer disodium cromoglicate, ${ }^{45}$ via inhibiting $\mathrm{MC}$ degranulation, or via in vitro experimental approaches. ${ }^{8,9}$ Therefore, this is the first report to explore the role of MCs in acupuncture and moxibustion analgesia using MC-deficient rats. Moreover, besides baseline mechanical and thermal pain thresholds, we also determined whether MC deficiency would influence the analgesic efficacy of acupuncture-and moxibustion-like interventions, and to what extent different stimuli modalities may affect mechanical and thermal pain analgesia using MCdeficient rats. Five different stimuli were randomly applied on different days mimicking acupuncture and moxibustion, at either innocuous $\left(43^{\circ} \mathrm{C}, 1 \mathrm{~mA}\right)$ or noxious $\left(46^{\circ} \mathrm{C}, 3 \mathrm{~mA}\right.$, MA) intensities. Our data clearly showed that mechanical nociception in WsRC-Ws/Ws rats could only be reduced by high-intensity stimuli. Indeed, this analgesic effect exists only when the stimulus intensity is strong enough (Figure 3), which is consistent with a previous study of acupuncture and moxibustion analgesia in normal rats. ${ }^{63}$ It has been confirmed by different research groups that high-intensity acupuncture and moxibustion stimuli over the $\mathrm{C}$-fiber threshold produce systemic antinociceptive effects at remote parts of the body from the pain site through diffuse noxious inhibitory control. ${ }^{64-66}$ Concerning the net changes evoked by different stimuli, the increases of MWL and MWT induced in WT rats were greater than in c-kit mutant rats (Figure 4). These data indicated that MC deficiency abolished the analgesic efficacy of low-intensity acupuncture- and moxibustion-like stimuli and attenuated the effect size of acupuncture and moxibustion at either low or high stimulation intensities (Figure 4).
Increasing studies have focused on the involvement of purinergic signaling in the pain pathway. ${ }^{67-69}$ It has also been reported that MCs are involved in acupuncture's analgesic effects via stimulating peripheral sensory nerves, affecting $\mathrm{Ca}^{2+}$ influx and ATP release. ${ }^{70}$ Previous studies described that the level of extracellular ATP concentration increased with MC degranulation and keratinocyte activation in response to mechanical and thermal stimulation. ${ }^{9,71,72}$ Extracellular ATP binding to the $\mathrm{P} 2 \mathrm{X}$ receptor located on primary afferent nerve endings, especially $\mathrm{P}_{2} \mathrm{X}_{3}$, would produce a painful sensation. However, extracellular ATP could usually not reach a concentration high enough to activate $\mathrm{P} 2 \mathrm{X}_{3}$ and other $\mathrm{P} 2 \mathrm{X}$ receptors. $^{71}$ This is because ATP is hydrolyzed by ecto-ATPases to AMP, the precursor of adenosine, which is a pivotal mediator for acupuncture analgesia via A1 receptor. ${ }^{71,73}$ In our study, WsRC-Ws/Ws rats were used for MC depletion owing to the c-kit gene deletion. ${ }^{25}$ Therefore, we suspect that less ATP is released because of fewer MCs responding to acupuncture and moxibustion intervention in the mechanical pain behavior tests. However, ATP is not only released by MCs, it may also be released by other cell types during mechanical stimulus. ${ }^{69}$ For this reason, we presume that MC deficiency did not abolish the analgesic effect induced by high-intensity acupuncture and moxibustion, only attenuating the analgesic efficacy in WsRC-Ws/Ws rats (Figure 4).

Interestingly, for thermal pain perception, both noxious and innocuous stimuli produced analgesic effects in both MCdeficient rats and their WT littermates (Figure 5). Further, no obvious differences in antinociceptive effects were observed in two animals except for $3 \mathrm{~mA}$ EA application (Figure 6). It is known that $\mathrm{P} 2 \mathrm{Y}_{2}$ receptors located on sensory endings participate in noxious heat sensation. ${ }^{74}$ Therefore, we believe that the $\mathrm{MC}$ deficit in mutant rats resulted in less ATP released at peripheral sites after the $46^{\circ} \mathrm{C}$ moxibustion-like stimulus, leading to less $\mathrm{P} 2 \mathrm{Y}_{2}$ receptor binding and activation, thereby influencing moxibustion analgesia. It is known that TRPV is the vital receptor for thermal nociception, ${ }^{60}$ and TRPV channel activation is involved in acupuncture, particularly in moxibustion analgesia. ${ }^{75}$ However, previous studies on the connection between MCs and TRPV were mainly focused on visceral hyperalgesia, ${ }^{26,42}$ and rare evidence has been published regarding somatic nociception.

\section{Limitation}

A limitation of the study is that we mainly observed changes in animals' nociceptive reflex behaviors before and after different acupuncture and moxibustion stimuli, which may have implications for the role of MCs in analgesia evaluated using 
the current gene deletion method. While previous investigations have offered biochemical labels and measurements of this line and because we are under a word-count constraint in the present manuscript, molecular and immunohistochemical studies will be reported in a future study to further characterize these effects.

\section{Conclusion}

This study defined the connection between MCs and acupuncture analgesia by using c-kit gene mutant rats. We demonstrated that MC deficiency elevated the baseline mechanical but not thermal pain threshold. Analgesic effects induced by innocuous acupuncture and moxibustion stimuli were eliminated in mechanical nociception, and effects induced by noxious stimuli were weakened in $\mathrm{MC}$ deficit rats. Otherwise, the net changes of analgesic efficacies were attenuated in WsRC-Ws/Ws rats. However, there were no differences in either the threshold or net changes in analgesic efficacy for thermal nociception in both genotypes. These data indicate that MCs are related to the antinociceptive efficacy of acupuncture- and moxibustion-like stimuli on mechanical pain in an intensity-dependent manner.

\section{Acknowledgments}

This study was supported by the National Natural Science Foundation of China grant to BZ (No. 81130063), XG (No. 81473778), and Youth Project of NSFC to KL (No. 81303054), The Fundamental Research Funds for the Central Public Welfare Research Institutes to BZ (No. ZZZD16001), and XG (No. GH2017-03-04). Thanks to Kristin Schoepfer for grammar polishing.

\section{Author contributions}

All authors contributed toward data analysis, drafting and revising the paper and agree to be accountable for all aspects of the work.

\section{Disclosure}

The authors report no conflicts of interest in this work.

\section{References}

1. Ernst E. Acupuncture therapy for neurological diseases. Focus on Altern Complement Ther. 2015;15(4):339-339.

2. Lu CY, Huang HC, Chang HH,et al. Acupuncture therapy and incidence of depression after stroke. Stroke. 2017;48(6):1682.

3. Radwan AM. Acupuncture and pain. Pain. 1984;18(Suppl 1):S151.

4. Yang L, Yang Z, Yu H, Song H. Acupuncture therapy is more effective than artificial tears for dry eye syndrome: evidence based on a metaanalysis. Evid Based Complement Alternat Med. 2015;2015:143858.
5. Han Z, Jiang YH, Wan Y, Wang Y, Chang JK, Han JS. Endomorphin-1 mediates $2 \mathrm{~Hz}$ but not $100 \mathrm{~Hz}$ electroacupuncture analgesia in the rat. Neurosci Lett. 1999;274(2):75-78.

6. Lin JG, Chen WL. Acupuncture analgesia: a review of its mechanisms of actions. Am J Chin Med. 2008;36(4):635-645.

7. Bing Z, Cesselin F, Bourgoin S, Clot AM, Hamon M, Le Bars D. Acupuncture-like stimulation induces a heterosegmental release of Metenkephalin-like material in the rat spinal cord. Pain. 1991;47(1):71-77.

8. Wang L, Lei H, Grygorczyk R, Shen X, Schwarz W. Modulation of extracellular ATP content of mast cells and DRG neurons by irradiation: studies on underlying mechanism of low-level-laser therapy. Mediators Inflamm. 2015;2015:630361.

9. Wang L, Sikora J, Hu L, Shen X, Grygorczyk R, Schwarz W. ATP release from mast cells by physical stimulation: a putative early step in activation of acupuncture points. Evid Based Complement Alternat Med. 2013;2013(2):350949.

10. Wu ML, Xu DS, Bai WZ, et al. Local cutaneous nerve terminal and mast cell responses to manual acupuncture in acupoint LI4 area of the rats. J Chem Neuroanat. 2015;68:14-21.

11. Gurish MF, Boyce JA. Mast cell growth, differentiation, and death. Clin Rev Allergy Immunol. 2002;22(2):107-118.

12. Florenzano F, Bentivoglio M. Degranulation, density, and distribution of mast cells in the rat thalamus: a light and electron microscopic study in basal conditions and after intracerebroventricular administration of nerve growth factor. J Comp Neurol. 2000;424(4):651-669.

13. Yong LC. The mast cell: origin, morphology, distribution, and function. Exp Toxicol Pathol. 1997;49(6):409-424.

14. Héron A, Dubayle D. A focus on mast cells and pain. J Neuroimmunol. 2013;264(1-2):1-7.

15. Anupam A, Afrin LB, Kalpna G. Mast cell-mediated mechanisms of nociception. Int J Mol Sci. 2015;16(12):29069-29092.

16. Anand P, Singh B, Jaggi AS, Singh N. Mast cells: an expanding pathophysiological role from allergy to other disorders. Naunyn Schmiedebergs Arch Pharmacol. 2012;385(7):657-670.

17. Wang L, Schwarz W. Activation of mast cells by acupuncture stimuli. For Immunopathol Dis Therap. 2012;3(1):41-50.

18. Shi Y, Qi L, Wang J, et al. Moxibustion activates mast cell degranulation at the ST25 in rats with colitis. World J Gastroenterol. 2011;17(32):3733-3738.

19. Johnson C, Huynh V, Hargrove L, et al. Inhibition of mast cell-derived histamine decreases human cholangiocarcinoma growth and differentiation via c-Kit/stem cell factor-dependent signaling. Am J Pathol. 2016;186(1):123-133.

20. Okada S, Kojima Y, Kubota Y, Mizuno K, Sasaki S, Kohri K. Attenuation of bladder overactivity in KIT mutant rats. BJU Int. 2011; 108(2 Pt 2):E97-E103.

21. Pittoni P, Piconese S, Tripodo C, Colombo MP. Tumor-intrinsic and -extrinsic roles of c-Kit: mast cells as the primary off-target of tyrosine kinase inhibitors. Oncogene. 2011;30(7):757-769.

22. Niwa Y, Kasugai T, Ohno K, et al. Anemia and mast cell depletion in mutant rats that are homozygous at "white spotting (Ws)" locus. Blood. 1991;78(8):1936-1941.

23. Tsujimura T, Hirota S, Nomura S, et al. Characterization of Ws mutant allele of rats: a 12-base deletion in tyrosine kinase domain of c-kit gene. Blood. 1991;78(8):1942-1946.

24. Bauer O, Razin E. Mast cell-nerve interactions. News Physiol Sci. 2000;15(5):213-218.

25. Kim YS, Lee MY, Ryu HS, et al. Regional differences in chronic stress-induced alterations in mast cell and protease-activated receptor2-positive cell numbers in the colon of Ws/Ws rats. J Neurogastroenterol Motil. 2014;20(1):54-63.

26. Yang CQ, Wei YY, Zhong CJ, Duan LP. Essential role of mast cells in the visceral hyperalgesia induced by $\mathrm{T}$. spiralis infection and stress in rats. Mediators Inflamm. 2012;2012:294070.

27. Tei H, Kasugai T, Tsujimura T, et al. Characterization of cultured mast cells derived from $\mathrm{Ws} / \mathrm{Ws}$ mast cell-deficient rats with a small deletion at tyrosine kinase domain of c-kit. Blood. 1994;83(4):916-925. 
28. Arizono N, Kasugai T, Yamada M, et al. Infection of nippostrongylus brasiliensis induces development of mucosal-type but not connective tissue-type mast cells in genetically mast cell-deficient $\mathrm{Ws} / \mathrm{Ws}$ rats. Blood. 1993;81(10):2572-2578.

29. Valent $P$. The riddle of the mast cell: kit(CD117)-ligand as the missing link? Immunol Today. 1994;15(3):111-114.

30. Bing Z, Villanueva L, Le Bars D. Acupuncture and diffuse noxious inhibitory controls: naloxone-reversible depression of activities of trigeminal convergent neurons. Neuroscience. 1990;37(3): 809-818.

31. Okada K, Oshima M, Kawakita K. Examination of the afferent fiber responsible for the suppression of jaw-opening reflex in heat, cold, and manual acupuncture stimulation in rats. Brain Res. 1996;740(1-2):201-207.

32. Zhu B, Xu WD, Rong PJ, Ben H, Gao XY. A C-fiber reflex inhibition induced by electroacupuncture with different intensities applied at homotopic and heterotopic acupoints in rats selectively destructive effects on myelinated and unmyelinated afferent fibers. Brain Res. 2004;1011(2):228-237.

33. Kagitani F, Uchida S, Hotta H, Aikawa Y. Manual acupuncture needle stimulation of the rat hindlimb activates groups I, II, III and IV single afferent nerve fibers in the dorsal spinal roots. Jpn J Physiol. 2005;55(3):149-155.

34. Kagitani F, Uchida S, Hotta H. Afferent nerve fibers and acupuncture. Auton Neurosci. 2010;157(1-2):2-8.

35. Kalmar B, Greensmith L, Malcangio M, McMahon SB, Csermely P, Burnstock G. The effect of treatment with BRX-220, a co-inducer of heat shock proteins, on sensory fibers of the rat following peripheral nerve injury. Exp Neurol. 2003;184(2):636-647.

36. Obata K, Yamanaka H, Kobayashi K, et al. Role of mitogen-activated protein kinase activation in injured and intact primary afferent neurons for mechanical and heat hypersensitivity after spinal nerve ligation. J Neurosci. 2004;24(45):10211-10222.

37. Lever I, Cunningham J, Grist J, Yip PK, Malcangio M. Release of BDNF and GABA in the dorsal horn of neuropathic rats. Eur $J$ Neurosci. 2003;18(5):1169-1174.

38. Glenner GG, Cohen LA. Histochemical demonstration of a speciesspecific trypsin-like enzyme in mast cells. Nature. 1960;185:846-847.

39. Schwartz LB, Metcalfe DD, Miller JS, Earl H, Sullivan T. Tryptase levels as an indicator of mast-cell activation in systemic anaphylaxis and mastocytosis. N Engl J Med. 1987;316(26):1622-1626.

40. Kelly RB. Acupuncture for pain. Am Fam Physician. 2009;80(5):481-484.

41. Kim HY, Wang J, Lee I, Kim HK, Chung K, Chung JM. Electroacupuncture suppresses capsaicin-induced secondary hyperalgesia through an endogenous spinal opioid mechanism. Pain. 2009;145(3):332-340.

42. Zou W, Lin H, Liu W, et al. Moxibustion relieves visceral hyperalgesia via inhibition of transient receptor potential vanilloid 1 (TRPV1) and heat shock protein (HSP) 70 expression in rat bone marrow cells. Acupunct Med. 2016;34(2):114-119.

43. Aich A, Afrin LB, Gupta K. Mast cell-mediated mechanisms of nociception. Int J Mol Sci. 2015;16(12):29069-29092.

44. Shi Y, Qi L, Wang J, et al. Moxibustion activates mast cell degranulation at the ST25 in rats with colitis. World J Gastroenterol. 2011;17(32): 3733-3738.

45. Zhang D, Ding G, Shen X, et al. Role of mast cells in acupuncture effect: a pilot study. Explore (NY). 2008;4(3):170-177.

46. Zhong A, Wu J, Hu Y. Study on correlation between the mast cell and the acupoint. World J Acupunct Moxibustion. 1994;4(4):53-58.

47. Maeda H, Yamagata A, Nishikawa S, et al. Requirement of c-kit for development of intestinal pacemaker system. Development. 1992;116(2):369-375.

48. Tsujimura T. Role of c-kit receptor tyrosine kinase in the development, survival and neoplastic transformation of mast cells. Pathol Int. 1996;46(12):933-938.

49. Galli SJ, Kitamura Y. Genetically mast-cell-deficient W/Wv and Sl/Sld mice. Their value for the analysis of the roles of mast cells in biologic responses in vivo. Am J Pathol. 1987;127(1):191-198.
50. Grimbaldeston MA, Chen CC, Piliponsky AM, Tsai M, Tam SY, Galli SJ. Mast cell-deficient W-sash c-kit mutant Kit W-sh/W-sh mice as a model for investigating mast cell biology in vivo. Am J Pathol. 2005;167(3):835-848.

51. Galli SJ, Nakae S, Tsai M. Mast cells in the development of adaptive immune responses. Nat Immunol. 2005;6(2):135-142.

52. Johnson D, Krenger W. Interactions of mast cells with the nervous system recent advances. Neurochem Res. 1992;17(9):939-951.

53. Schwartz LB, Austen KF. Enzymes of the mast cell granule. J Invest Dermatol. 1980;74(5):349-353.

54. Matsuda H, Kawakita K, Kiso Y, Nakano T, Kitamura Y. Substance P induces granulocyte infiltration through degranulation of mast cells. J Immunol. 1989;142(3):927-931.

55. Li WW, Guo TZ, Liang DY, Sun Y, Kingery WS, Clark JD. Substance $P$ signaling controls mast cell activation, degranulation, and nociceptive sensitization in a rat fracture model of complex regional pain syndrome. Anesthesiology. 2012;116(4):882-895.

56. Koda H, Mizumura K. Sensitization to mechanical stimulation by inflammatory mediators and by mild burn in canine visceral nociceptors in vitro. J Neurophysiol. 2002;87(4):2043-2051.

57. Yu S, Kollarik M, Ouyang A, Myers AC, Undem BJ. Mast cellmediated long-lasting increases in excitability of vagal $\mathrm{C}$ fibers in guinea pig esophagus. Am J Physiol Gastrointest Liver Physiol. 2007;293(4):G850-G856

58. Steinhoff M, Vergnolle N, Young SH, et al. Agonists of proteinaseactivated receptor 2 induce inflammation by a neurogenic mechanism. Nat Med. 2000;6(2):151-158.

59. Benham CD, Gunthorpe MJ, Davis JB. TRPV channels as temperature sensors. Cell Calcium. 2003;33(5-6):479-487.

60. Julius D. TRP channels and pain. Annu Rev Cell Dev Biol. 2013;29: 355-384.

61. Mogil JS, Wilson SG, Chesler EJ, et al. The melanocortin-1 receptor gene mediates female-specific mechanisms of analgesia in mice and humans. Proc Natl Acad Sci U S A. 2003;100(8):4867-4872.

62. Delaney A, Keighren M, Fleetwood-Walker SM, Jackson IJ. Involvement of the melanocortin-1 receptor in acute pain and pain of inflammatory but not neuropathic origin. PLoS One. 2010;5(9):e12498.

63. Xin JJ, Su YS, Yang ZK, et al. [Effects of electroacupuncture and regional thermal stimulation at "Zusanli" (ST 36) on pain thresholds of TRPV 1 knock-out mice]. Zhen Ci Yan Jiu. 2012;37(6): 431-439. Chinese.

64. Murase K, Kawakita K. Diffuse noxious inhibitory controls in antinociception produced by acupuncture and moxibustion on trigeminal caudalis neurons in rats. Jpn J Physiol. 2000;50(1):133-140.

65. Sims J. The mechanism of acupuncture analgesia: a review. Complement Ther Med. 1997;5(2):102-111.

66. Liu J, Fu W, Yi W, et al. Extrasegmental analgesia of heterotopic electroacupuncture stimulation on visceral pain rats. Brain Res. 2011;1373(3): $160-171$.

67. Abbracchio MP, Burnstock G, Verkhratsky A, Zimmermann H. Purinergic signalling in the nervous system: an overview. Trends Neurosci. 2009;32(1):19-29.

68. Burnstock G. Acupuncture: a novel hypothesis for the involvement of purinergic signalling. Med Hypotheses. 2009;73(4):470-472.

69. Burnstock G. Purinergic receptors and pain. Curr Pharm Des. 2009; 15(15):1717-1735

70. Yao W, Yang H, Yin N, Ding G. Mast cell-nerve cell interaction at acupoint: modeling mechanotransduction pathway induced by acupuncture. Int J Biol Sci. 2014;10(5):511-519.

71. Goldman N, Chen M, Fujita T, et al. Adenosine A1 receptors mediate local anti-nociceptive effects of acupuncture. Nat Neurosci. 2010;13(7):883-888.

72. Praetorius HA, Leipziger J. ATP release from non-excitable cells. Purinergic Signal. 2009;5(4):433-446.

73. Zylka MJ. Pain-relieving prospects for adenosine receptors and ectonucleotidases. Trends Mol Med. 2011;17(4):188-196. 
74. Malin SA, Davis BM, Koerber HR, Reynolds IJ, Albers KM, Molliver DC. Thermal nociception and TRPV1 function are attenuated in mice lacking the nucleotide receptor P2Y2. Pain. 2008;138(3):484-496.
75. Jiang J, Wang X, Wu X, Yu Z. Analysis of factors influencing moxibustion efficacy by affecting heat-activated transient receptor potential vanilloid channels. $J$ Tradit Chin Med. 2016;36(2):255-260.

\section{Publish your work in this journal}

The Journal of Pain Research is an international, peer reviewed, open access, online journal that welcomes laboratory and clinical findings in the fields of pain research and the prevention and management of pain. Original research, reviews, symposium reports, hypothesis formation and commentaries are all considered for publication
The manuscript management system is completely online and includes a very quick and fair peer-review system, which is all easy to use. Visit http://www.dovepress.com/testimonials.php to read real quotes from published authors. 